\title{
Rodents move towards the Disease Ontology
}

\author{
Ellen P. Neff
}

W hat exactly is a disease?

Definitions and criteria are often in flux, but resources are being updated to help make sure everyone's speaking the same language, at least when moving between humans, mice, and rats.

Earlier this year, collaborators representing the three species published an overview of a three-year project to review and align disease terms in their respective databases with the Disease Ontology (DO), a project that's been funded by the National Human Genome Research Institute as a Genomic Resource to provide a standardized ontology of human disease for use by the biomedical community. An overview of the collaboration can be found in Disease Models and Mechanisms (https://doi.org/10.1242/dmm.032839).

The curators at DO were used to working with the rodent groups to review and add terms in the past, but "this was a funded effort to really harmonize the data between the mouse and the rat groups and to do it through the Disease Ontology," says DO's principal investigator

Lynn Schriml. DO was already used by numerous clinical and preclinical groups; by adopting it, information from the Mouse Genome Database (MGD) and Rat Genome Database (RGD) now has the same links across communities.

Those linkages include Online Mendelian Inheritance in Man (OMIM), a vocabulary source that MGD relied heavily on in the past. While a valuable vocabulary describing genetically inherited diseases, OMIM is not actually an ontology that defines hierarchical relationships. It also lacks generic disease concepts; rather, diseases without any known genetic mutations are considered "undefined." That was sufficient for a time, but improving genetics have been taking MGD somewhat backwards.

"It was sort of reaching a crisis point with OMIM because as we were getting more and more genetic information for diseases, we were losing more and more of these undefined entries," explains Susan Bello, lead author and Senior Scientific Curator at Mouse Genome Informatics (MGI), the umbrella term for several projects, including MGD, that are accessed via www.informatics.jax.org. Take, for example,
Inflammatory Bowel disease (IBD). When OMIM:266600, an initially undefined IBD entry, was redefined as "Inflammatory bowel disease 1," just one mouse model remained appropriate. Twenty three other annotations had to be moved to the 'notes' field in MGI: still available, but not as easily searchable through the website's tools.

The DO fit the criteria they were looking for in an alternative source: broad in scope with hierarchical, stable terms for both generic and highly specific diseases. All while still enabling cross-linking to OMIM terms and others, like Orphanet (a rare disease vocabulary) and Medical Subject Heading (MeSH) terms, where applicable.

The collaboration's deep dive across their resources involved several in-person meetings, regular conference calls, and many spreadsheets where Bello and her RGD counterpart, Stanley Laulederkind, reviewed their terms and compared them against those in the DO, making recommendations for new and updated terms and crosslinking existing ones along the way. The DO was updated throughout the process-all updates are tracked and history is available online-while MGI switched its front end display on March 13, 2017 and its backend annotation tools a few months later on June 5.

Though mice are fully converted to the DO, rats remain on somewhat of a hybrid system. Disease terms there are mapped to the ontology wherever possible, allowing rat and mouse (and beyond) to be interlinked, but rats still have quite a bit extra.

Researchers using rats are often looking well beyond genetics: biomarkers, injury models, experimentally induced conditions, etc-things that don't always map neatly to a genetic disease. Whereas prior to the project RGD used a mix of MEDIC, itself a merged vocabulary of OMIM and MeSH terms, with their own RGD-developed disease ontology, the DO has now quietly replaced the MEDIC portions.

Though there are still plenty of terms Laulederkind would like to see added to DO (particularly related to non-genetic data and models that could have relevance to human clinicians and other model organism researchers), the ontology did make some structural updates to better serve the rodent communities. Previously, it was structured based on etiology, but that's not the only way researchers may think about a particular disease. An alternative anatomy-based tree was also built through the collaboration. It's available in MGI, and in progress for visualization at DO's website. "Getting the anatomy in there has been a really big help and I think it will be far more useful for our users that way," says Bello.

As of the March publication, over 1200 new terms, 1900 cross-references, and 1100 new relationships were added to the DO as a result of the collaboration with MGD and RDG. And the DO remains a living project. As new data emerges, so will new disease terms and relationships. A more complex disease model is also in the works, and the first models are anticipated for the public within the year says Schriml. A team of clinicians at the University of Maryland School of Medicine is currently breaking down an initial set of complex diseases, such as Prader-Willi syndrome, into their different genotype-phenotype views; having links to the model organism databases should help researchers then follow-up on those complexities, she hopes.

The adoption of DO helps ensure that mice and rats are using consistent terms and relationships wherever possible, but that will extend to other model organism groups as well: the Alliance of Genome Resources will also be relying on DO. The Alliance is a consortium established in 2016 to better integrate data between six different model organism databases: MGD; RGD; Drosophila researchers' FlyBase; WormBase, for C. elegans data; ZFIN, the Zebrafish Information Network; and, leaving the realm of animals, the Saccharomyces Genome Database. "If all the models are using a common vocabulary, then at least you only have to learn one vocabulary," says Bello. "Anybody who is doing disease-related work, it should make their lives easier."

Ellen P. Neff

Lab Animal.

e-mail:Ellen.neff@us.nature.com

Published online: 23 May 2018

https://doi.org/10.1038/s41684-018-0073-0 